Article

\title{
Outcomes of food handlers' medical examinations conducted at an occupational health clinic in Zimbabwe
}

\author{
*Dingani Moyo ${ }^{1,2,3}$, Florence Moyo ${ }^{3 ; 4}$ \\ ${ }^{1}$ University of the Witwatersrand, School of Public Health, Faculty of Health Sciences, Occupational Health \\ Division, Johannesburg, South Africa. moyod@iwayafrica.co.zw and dingani.moyo@wits.ac.za
}

${ }^{2}$ Midlands State University, Faculty of Social Sciences, Gweru, Zimbabwe

${ }^{3}$ Baines Occupational Health services, 27 Baines Avenue, Harare, Zimbabwe

${ }^{4}$ Zimbabwe Open University, Faculty of Sports and Science. 6 Victory Road, Gweru, Zimbabwe. flormoyo@iwayafrica.co.zw

*Correspondence: moyod@iwayafrica.co.zw and dingani.moyo@wits.ac.zaTel.: +263 772150115

\begin{abstract}
Food handlers' medical examinations are mandated by most countries as a way of safeguarding the health and safety of consumers. Food-borne diseases are an important cause of morbidity and mortality. In Zimbabwe, the use of chest radiographs and throat and rectal swab tests are a requirement during food handlers' medical examinations. This study aimed at exploring the patterns and outcomes of physical medical examinations, chest radiographs, and other tests of food handlers. A cross-sectional review of retrospective occupational health records was carried out. The mean age for the study population was 37 years with an age range of 21 to 56 years. Males accounted for $73 \%$ of the study participants. All of the 157 rectal swabs were normal and did not culture any organism. Fifteen percent (24) of the throat swabs cultured one or more organisms. Ninety-seven percent of chest radiographs were normal. Ninety-seven percent of employees were certified as fit. Thirty-six percent of the food handlers were in the overweight and obese categories. Hypertension and high blood pressure were common conditions in the study sample. It can be concluded that routine radiological and laboratory testing of the food handlers in this study was of little value.
\end{abstract}

Keywords: food handlers; medical examinations; fitness; radiology; rectal and throat swabs

\section{Introduction}

According to the Centers for Disease Control (CDC), as cited in Mathew RR (2019), 20 to 40\% of food borne illness associated with the consumption of contaminated food originated in catering establishments [1]. According to the World Health Organization (WHO; 2015), foodborne diseases are an important cause of morbidity and mortality, and a significant impediment to socioeconomic development globally [2]. Furthermore, the WHO states that $43 \%$ of the disease burden from contaminated food occurred in the under 5 years age group, (WHO, 2015). Asia and Sub-Saharan Africa have the highest incidence of foodborne diseases. Unsafe food costs low-and middle-income economies USD 110 billion in lost productivity and medical expenses each year [3]. One in ten people fall ill after eating contaminated food globally (WHO, 2015).

In 1922, Craster, C.V. described the requirements for a complete set of food handler examinations as a thorough chest examination to be made for tuberculosis, an examination for skin diseases or eruptions, a routine throat swab, a Widal test for typhoid fever, and, in males, the examination of the external genitals for venereal diseases [4]. Such an examination would provide 
the minimum safeguards against infection by food. On 27 March 1927, M. James Fine deemed it unwise to continue with the practice of taking nose and throat cultures after none of the 27,000 specimens tested positive [5]. This practice was subsequently stopped in July 1927 and emphasis was given to physical examination of the throat [5].

Biswal Manisha et al. (2012) found that the prevalence of enteric organisms in routine examinations of food handlers was low at 6.5\% [6]. They recommended regular training on food safety and emphasis on self-reporting of diarrhea symptoms as being key in food safety. Routine testing of food handlers feces, blood or rectal swabs is neither cost effective nor generally warranted [7].

It has long been established that conducting preplacement examinations and routine periodic medical examinations, inclusive of investigations of stool or rectal swabs, throat swabs, blood tests, and chest X-rays, is of no value and should not be carried out [8]. The emphasis, however, is on assessments of food handlers who are unwell.

In a study of 300 food handlers working in hotels in Ethiopia, Getenet B. et al. (2019) found that the overall prevalence of staphylococcus in the nasal carriage was 9\% [9]. They recommended the need for protective measures including increased public awareness programs, regular monitoring of food handlers for food borne pathogens, and intensive training on primary health care and hygiene. In a study of 151 food handlers in New Delhi, Malhotra R. et al. (2007) found that most (94.7\%) had one or more morbid conditions, with the important ones being dental caries $(50.3 \%)$, worm infestation $(41.1 \%)$, and injuries/burns on hands (39.7\%) [10]. They noted that health-seeking behavior, immunization status in relation to the enteric group of fevers and tetanus, and pre-placement and inservice medical examinations were observed to be unsatisfactory.

Kamal Paul et. al (2012) found that the parasites Ascaris lumbricoides, Entamoeba histolytica, and Giardia lamblia were observed in certified food-handlers [11]. Ranl Rose Mathew et al (2019), in a study of 123 study participants in India, found a prevalence of enteric pathogens of $5.2 \%$, in which four stool specimens were positive for intestinal parasites (two-Giardia intestinalis, one-Entamoeba histolytica, one-Blastocystis hominis) and Campylobacter jejuni was isolated from stool samples of two food handlers [12]

From a total of 212 microbiological samples, Metin Erdogan and Şebnem Pamuk (2019) found that most isolates were harvested from hands $(52 \%)$ and food samples $(36 \%)$, whereas few isolates $(12 \%)$ were obtained from kitchen equipment [13]. Ncube, F. et al (2020) found that data from field observations indicated that contrary to self-reported information, a substantial proportion of food handlers did not use a detergent or disinfectant to wash their hands before food handling or posthandling potentially contaminated materials [14]. About half of the food handlers washed their hands in sinks meant for washing cutlery, $57 \%$ did not use approved hand drying methods, and $19.8 \%$ did not adequately thaw frozen foods. Coli et. al (2014) found that parasitic infections were observed in $28 \%(42 / 150)$ of street food vendors that responded to the questionnaire in Brazil [15].

The main objective of the current study was to profile the outcomes of food handlers' medical examinations in Zimbabwe. Furthermore, this study sought to establish the main organisms prevalent in throat and rectal swabs. The study also sought to establish the usefulness of radiological and laboratory investigations in food handlers' medical examinations.

\section{Materials and Methods}

A retrospective records review was conducted for two companies whose employees underwent food handlers' medical examinations at a specialist occupational health clinic in Harare, Zimbabwe. One company had 99 food handlers while the other had 58. All food handlers' medical records that included a doctor's physical examination, chest X-ray findings, and throat and rectal swab examinations were included in the study. Both companies were involved in the manufacture of food stuffs. The food handlers' medical examinations were performed at two different times of the year in 
2019 and 2020, six months apart, and each company conducted chest X-rays and laboratory investigations at different service providers. Ethical approval for this research was obtained from the Medical Research Council of Zimbabwe, MRCZ- E/268. Data was collected and analyzed in Microsoft Excel 2016 version and presented in graphs and tables. Data analysis was categorized by fitness outcomes, radiological and laboratory testing, and physical examination findings.

\section{Results}

\subsection{Demographics}

A total of 157 workers from two different companies underwent food handlers' medical examinations. The mean age for the study population was 37 years and $73 \%$ of the study participants were male. One of the companies had a total of 99 employees with a mean age of 39 years and an age range of 25 to 56 years. In this group, males constituted $64 \%$ (65) and females $36 \%$ (34). The second company had 58 employees with a mean age of 36 years and an age range of 21 to 50 years and $86 \%$ were males

\subsection{Radiological Findings}

Ninety-seven percent (152) of the X-rays were normal while only 3\% (5) were abnormal. The abnormal chest radiographs were for patients who had been previously treated for tuberculosis or were current cardiac patients with renal failure, and three were patients suffering from hypertension. All of the patients with abnormal findings from X-rays were certified fit as they did not have any evidence of a disabling condition or infectious disease.

\subsection{Laboratory findings: Rectal swabs and throat swabs}

All (157) of the rectal swabs were normal and did not culture any organism. Of the 157 throat swabs that were conducted, $15 \%$ (24) cultured one or more of the following organisms: Klebsiella, Candida albicans, Pseudomonas aeruginosa, or non-lactose fermenting coliforms, as shown in Table 1. Of the 24 throat swab specimens, the commonest organism that was cultured was Klebsiella with a frequency of $24 \%(11)$

Table 1: Throat swab results with growths. $n=24$

\begin{tabular}{|r|l|c|r|}
\hline & Name of organosm cultured & Frequency & $\%$ \\
\hline 1 & Klebsiella & 11 & $45.8 \%$ \\
\hline 2 & Candida albicans & 5 & $20.8 \%$ \\
\hline 3 & Pseudomonas aeruginosa & 1 & $4.2 \%$ \\
\hline 4 & Non lactose fermenting coliforms & 7 & $29.2 \%$ \\
\hline
\end{tabular}

\subsection{Fitness for duty outcomes}


Only 3\% (5) of the workers were certified as temporarily unfit with three having poorly controlled blood pressure and two having poor vision. None was certified as permanently unfit to work.

\subsection{Blood pressure and BMI outcomes}

Overall, $6 \%$ (10) of the workers were known hypertensives and 10\% (16) had an elevated blood pressure. Hence, $16 \%$ of the food handlers had either raised blood pressure or were known hypertensive patients. From the 99 food handlers with a recorded BMI, 37\% (37) were in the overweight and obese categories. Of the 18 participants who either were known hypertensives or had a raised blood pressure, 39\% (7) had a BMI of 25 and above. Of the 99 food handlers with a recorded medical history, seven were known hypertensives receiving treatment and nine reported to be suffering from eye problems. Elevated blood pressure and poor near point vision were the commonest medical findings, with frequencies of $17 \%$ and $12 \%$, respectively.

Table 2: Common medical examination findings.

\begin{tabular}{|l|l|l|}
\hline & Medical Condition & Frequency (\%) $\quad$ N = 157 \\
\hline 1 & Hypertensives receiving treatment & $10(6.5 \%)$ \\
\hline 2 & Raised blood pressure & $16(10 \%)$ \\
\hline 4 & Poor near point vision & $18(12 \%)$ \\
\hline 5 & Other conditions & $13(8.5 \%)$ \\
\hline & & \\
\hline
\end{tabular}

Of the 99 workers who had recorded BMIs, the mean age was 39 years with an age range of 2556 years. Males constituted $65 \%$ while $35 \%$ were females. The mean length of employment was 10 years. Eighteen (18\%) of workers were underweight, 45 (46\%) had a normal BMI, $24(24 \%)$ were overweight, and $12(12 \%)$ were obese.

\section{Discussion}

Our study findings reveal that all the rectal swabs were normal, and no organisms were isolated in the cultures. This finding contrasts with those of Awol, N et. al. 2019 who found that $2.12 \%$ were positive for salmonella from a total of 236 study participants [16]. Our findings support the current practice that does not recommend routine rectal swabs of food handlers except in cases where there is an indication [8]). The yields from the throat swabs were low, with Klebsiella being the commonest organism cultured. Our findings are similar to those of Biswal Manisha et al. (2012) and Ranl Rose Mathew et al. (2019), who found that the prevalence of enteric organisms in routine examinations of food handlers was quite low [6;12]. Overall, our findings for both rectal and throat swabs reveal that there is no value added by conducting such tests as part of routine food handlers' medical examinations.

Chest radiographs were predominantly normal with a small number yielding findings of old tuberculosis and cardiac problems. This finding goes against the routine performance of radiographs during food handlers' medical examinations, and the provisions of the Public Health Act of Zimbabwe and by-laws of the local authorities in Zimbabwe. An extremely low proportion of the examinations were certified as unfit for duty during the physical examinations. Pachman 2009 asserts that routine medical examinations have long since been discounted, although in Zimbabwe these are mandated. Routine medical examinations have been shown not to add any significant value [17]. Our findings show that there was no added value in performing routine physical examinations, and stool and throat swab examinations, on food handlers. 
A significant proportion of food handlers were in the overweight and obese categories. These findings concur with those of Roberto De Vogli et al (2014) who found that fast food consumption is independently and positively associated with obesity [18].

Most of the workers who were either known hypertensives or had an elevated blood pressure had BMIs above 25. Overweight and obesity have been demonstrated to be associated with an increase in the prevalence of lifestyle diseases such as hypertension and diabetes. Workplace wellness programs are increasingly being recommended to manage lifestyle diseases in workplaces.

Hypertension and elevated blood pressure were the most prevalent conditions that were identified. Visual problems constituted the second most common condition that was identified among the food handlers. Screening for visual problems and high blood pressure should be prioritized during routine medical examinations for food handlers.

This study was limited to a small sample size and cannot be generalized for the entire food industry. However, despite its small sample size, the study's findings are consistent with other research. Being a cross-sectional study, the study has limitations of establishing causality of the observed medical conditions. Retrospective record reviews are often limited by incomplete data and can only reflect the burden of the conditions under study.

\section{Conclusions}

It can be concluded from the study that performing routine physical medical examinations, chest radiographs, and throat and rectal swabs probably does not add value, as evidenced by the insignificant yields of these examinations. It is also clear from the study that hypertension and raised blood pressure are significant conditions within the food industry. It is recommended that food handlers' health surveillance be anchored on good hygiene practices and focused on screening for common medical conditions. Based on the findings of this study, we make the following recommendations:

1. The Public Health Act of Zimbabwe and by-laws of local authorities should be amended to align to current occupational health practices for food handlers.

2. Chest radiographs, and throat and rectal swabs should not be done as a perfunctory routine but only when indicated.

3. In the challenging economic environment currently facing Zimbabwe, the adoption of comprehensive questionnaire-based screening for food handlers is recommended. Full physical examinations and other tests should be dictated by other indications.

4. Organizations should set up wellness programs to promote nutrition and other key health needs for food handlers.

5. Screening for common health conditions, such as hypertension and visual problems, should be an integral part of surveillance programs.

Author Contributions: Dingani Moyo conceptualized and prepared the manuscript, and Florence Moyo developed the methodology for data analysis. Dingani Moyo and Florence Moyo analysed the data and assisted in the editing of the manuscript. Florence Moyo edited the manuscript and validated the methodology for data collection and analysis.

Funding: This research received no external funding

Acknowledgments: We would like to acknowledge the administrative support offered by Hellen Masvingo in the preparation of the manuscript.

Conflicts of Interest: The authors declare no conflict of interest.

\section{References}


1. Mathew RR, Ponnambath DK, Mandal J, Roy G. Enteric Pathogen Profile and Knowledge, Attitude and Behavior about Food Hygiene among Food Handlers in a Tertiary Health Care Center. Int J Med Public Health. 2019;9(3):60-5.

2. World Health Organization 2015. WHO Estimates of the Global Burden of Food borne diseases. Foodborne Disease Burden Epidemiology Reference Group $2007 \quad-2015$. https://apps.who.int/iris/bitstream/handle/10665/199350/9789241565165 eng.pdf?sequence=1 Accessed 25 April 2020.

3. The World Bank, 2018. Understanding Poverty. The Safe Food Imperative: Accelerating Progress in Low- and Middle-Income Countries.

https://www.worldbank.org/en/topic/agriculture/publication/the-safe-food-imperative-acceleratingprogress-in-low-and-middle-income-countries Accessed 25 April 2020

4. Craster, C.V 1922. The Medical Examination of Food Handlers. The American Journal of Public Health.

5. M. James Fine 1927. Examination of Food Handlers: Public Health Reports (1896-1970), Vol. 42, No. 12 (Mar. 25, 1927), pp. 799-804 Published by: Sage Publications, Inc. Stable URL: https://www.jstor.org/stable/4578238

6. Biswal Manisha et al. (2012). Is Routine Medical Examination of Food Handlers Enough to Ensure Food Safety in Hospitals? The Journal of communicable diseases 44(3):139-44

7. International Federation of Infection Control. Basic Concepts of Infection Control. Food Hygiene. IFIC: 2008. http://api.ning.com/files/P9gZGBaPyWeUGII1YwpAVMYnM-

PFun1LetUNYIun50MOJhVwdmwVklTrCjNk6w-I1Exu291AreEHRHQJv-jKEQuYxqDdwJA/foodhygiene1.pdf Accessed 24 April 2020

8. WHO Consultation on Health Surveillance and Management Procedures for Food-Handling Personnel (1989: Geneva) \& World Health Organization. (1989). Health surveillance and management procedures for food-handling personnel: Report of a WHO consultation [held in Geneva from 18 to 22 April 1988]. World Health Organization. https://apps.who.int/iris/handle/10665/39610

9. Getenet Beyene, Girma Mamo, Tesfaye Kassa, Getnet Tasew, Seid Tiku Mereta. Nasal and Hand Carriage Rate of Staphylococcus aureus among Food Handlers Working in Jimma Town, Southwest Ethiopia. Ethiop J Health Sci.2019;29(5):605.doi: http://dx.doi.org/10.4314/ejhs.v29i5.11

10. Malhotra Rahul, Lal Panna, Krishna Prakash, S. Daga, Mridul K. Kishore, Juga Profile of food handlers working in food service establishments located within the premises of a medical college in Delhi, India. In Public Health 2007 121(6):455-461. https://doi.org/10.1016/j.puhe.2006.08.002

11. Kamau,Paul, Aloo-Obudho, Penina, Kabiru Ephantus, Ombacho Kepha, Langat Bernard, Mucheru Obadiah, Ireri Laban. Prevalence of intestinal parasitic infections in certified food-handlers working in food establishments in the City of Nairobi, Kenya. Journal of Biomedical Research March 2012 26(2):84-89. https://doi.org/10.1016/s1674-8301(12)60016-5

12. Rani Rose Mathew, Dinoop Korol Ponnambath, Jharna Mandal, Gautam Roy. Enteric Pathogen Profile and Knowledge, Attitude and Behavior about Food Hygiene among Food Handlers in a Tertiary Health Care Center. Int J Med. Public Health. 2019;9(3):60-6.

13. Metin Erdogan and Şebnem Pamuk, (2020). Microbial contamination in food, food- handlers' hands and surfaces and evaluation of contamination sources by the similarity between isolates. Ankara Üniv Vet Fak Derg, 67, 73-79, 2020 DOI:10.33988/ auvfd.59936

14. Ncube France, Kanda Artwell, Chijokwe Morleen, Mabaya Goden, Nyamugure Tendayi, 2020. Food Science \& Nutrition; Mar2020, Vol. 8 Issue 3, p1677-1687, 11p

15. Colli, Cristiane Maria, Mizutani, Angelica Sayuri; Martins, Vanessa Aparecida, Ferreira, Érika Cristina, Gomes, Mônica Lúcia. Prevalence and risk factors for intestinal parasites in food handlers, southern Brazil. International Journal of Environmental Health Research. Sep2014, Vol. 24 Issue 5, p450-458. 9p. 4 Charts. 
16. Neja Awol, Demiss Nigusse, and Musa Ali, 2019. Prevalence and antimicrobial susceptibility profile of Salmonella and Shigella among food handlers working in food establishment at Hawassa city, Southern Ethiopia. BMC Res Notes (2019) 12:712 https://doi.org/10.1186/s13104-019-4725-y

17. Pachman, J. (2009). Evidence base for pre-employment medical screening. Bulletin of the World Health Organization, 87, 529-534. doi:10.2471/BLT.08.052605

18. Roberto De Vogli,a Anne Kouvonenb \& David Gimeno. Bull World Health Organ 2014;92:99-107A । doi: http://dx.doi.org/10.2471/BLT.13.120287 